\title{
A CASE OF MUTUAL REDUCTION OF DOMINANCE : OBSERVED IN MUS MUSCULUS
}

\author{
MARGARET E. WALLACE \\ Department of Genetics, Cambridge
}

Received 30.vi.52

A DOMINANGE interaction is already known between genes at the normal/pink-eyed-dilution locus $(P / p)$ and the black/brown locus $(B / b)$ in Mus musculus. Snell (193I) reports " Pink-eyed non-agouti mice carrying brown in the heterozygous condition are distinguishable from those homozygous for black by their lighter shade." That is, $p p$ is a dominance modifier of $B$.

In the stocks under my observation in this department, it now appears that $b b$ is also a dominance modifier of $P$; for brown nonagouti mice heterozygous for pink-eye are distinguishable from those homozygous for non-pink-eye.

That is, there is a mutual reduction of dominance at these two loci. A table of the six distinguishable phenotypes is given below :-

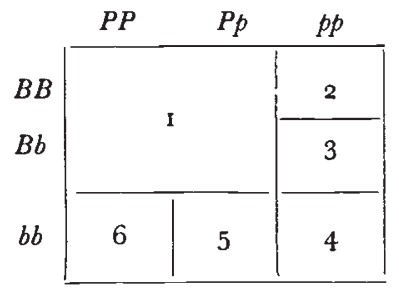

This phenomenon was first observed as a segregation of "pale" and "non-pale" among browns. Routine genetic tests were then set up which established

(i) the dominance of " pale" in bbaa genotypes;

(ii) that single-factor ratios were obtained in all kinds of matings involving " pale";

(iii) that the " pink-eyes " produced from intercrosses of " pales," and from backcrosses of "pale" to "pink-eyes" were $p p$ and not papa (pallid) ; and that

(iv) "pales" could be produced at will by crossing ppbbaa $\times P P b b a a$.

In the stocks used for outcrosses and identity tests $P p$ was usually found to be "pale" ; but classification in these and the other matings was not always quite certain, and it may be possible to find stocks in which Ppbbaa is indistinguishable from PPbbaa.

The nine genotypes involving the $p$ and $b$ loci were made up, and several specimens of each were skinned. This work confirmed Snell's (193I) findings that $B b p p$ were distinguishable from $B B p p$. 
About five skins were made of each of the genotypes 2, 3, 5 and 6 of the table. In these there was observed some phenotypic overlapping of genotypes 2 and 3, and of genotypes 5 and 6 . Thus, it appears that the degree of reduction of dominance between these two loci is itself dependent on other modifying factors. It is possible, therefore, that selection in a suitably heterogeneous stock could establish complete dominance at either locus. All the genotypes in class I of the table appeared to have the same intensity of pigmentation; but a larger experiment might reveal very small differences.

Two sets of skins of the nine genotypes have been made into an exhibit : one will be kept in this department and the other is in the Genetics Laboratory, Oxford. In each case, the skin selected for the exhibit, from among a set of one genotype, was that judged by eye to be median in intensity of colour for that genotype. A blackand-white photograph of the nine skins, selected in the same way from all the skins available, appears in plate $\mathrm{I}$. This gives some idea of the contrasts observable but naturally does not show them as satisfactorily as would a colour reproduction. Several attempts were made in colour, but faithful reproduction of hue as well of intensity was found to be too difficult.

From the skins it can be seen that the ratio of the intensity of colour of the genotypes $3: 2$ and $5: 6$ is about the same. That is, the action of the homozygote at each locus on the heterozygote at the other locus is roughly equivalent. It seems probable, on this evidence, that the mutant alleles at the two loci have reached about the same stage in their evolution.

On Fisher's (193I) theory of the evolution of dominance, three main factors govern the rate of evolution of a mutant at a particular locus :-

(i) the date at which the mutation first occurred at that locus.

(It must be remembered that this may ante-date the differentiation of the species, often even of the genus and of higher orders) ;

(ii) the initial and subsequent viability primarily of the heterozygote and secondarily of the homozygote; and

(iii) the subsequent mutation-rate.

It should in theory be possible to deduce any of these factors, from knowledge of the other factors and of the present interactions of mutants. As more knowledge is acquired on these points, it should become possible to make certain conclusions and to make them with increasing validity. Such knowledge will derive from various sources. Comparisons of the actions and reactions of mutants within different genera, orders and classes etc., gives a lower limit to the age of such mutants (Fisher, ibid., p. 35I). Initial and subsequent viabilities cannot be brought under direct observation, but fossil histories, combined with a study of the present viability of mutants on various artificially produced genetic environments, may help to give a general 
idea of the initial kinds and degrees of effects of mutants which are still occurring. A history of mutation-rates is also not readily accessible. That these rates may not always have been the same has been pointed out by Fisher : "It is probable . . . that the genetic changes which have brought about evolutionary transformation of species have been accompanied by corresponding changes in the frequency and kind of deleterious mutations to which their germplasms are prone." This possibility for particular loci may also become capable of verification, with the aid of increased palæontological knowledge, and by a study of present mutation rates which do come under immediate observation.

A tentative speculation may thus be made about the evolutionary age of $p$ and $b$ in Mus musculus. Making the following assumptions, which may become verifiable, that $p$ and $b$ had a similar history of mutation-rate and similar initial viability, it would not be inconsistent to conclude that the two mutants first occurred at about the same period of evolution; that is that they are about the same age.

I am indebted to Professor Sir Ronald Fisher, F.R.s., for advice and criticism of this paper, and to Miss M. A. C. Vincent for skinning some fifty specimens. I would also like to thank Messrs Oliver and Boyd for their attempts to obtain faithful colour reproductions of the skins.

\section{REFERENCES}

FISHER, R. A. 1931. The evolution of dominance. Biological Reviews, 6, No. I, 345-368.

SNELL, G. D. 1931. Inheritance in the house mouse. Genetics, $16,42$. 


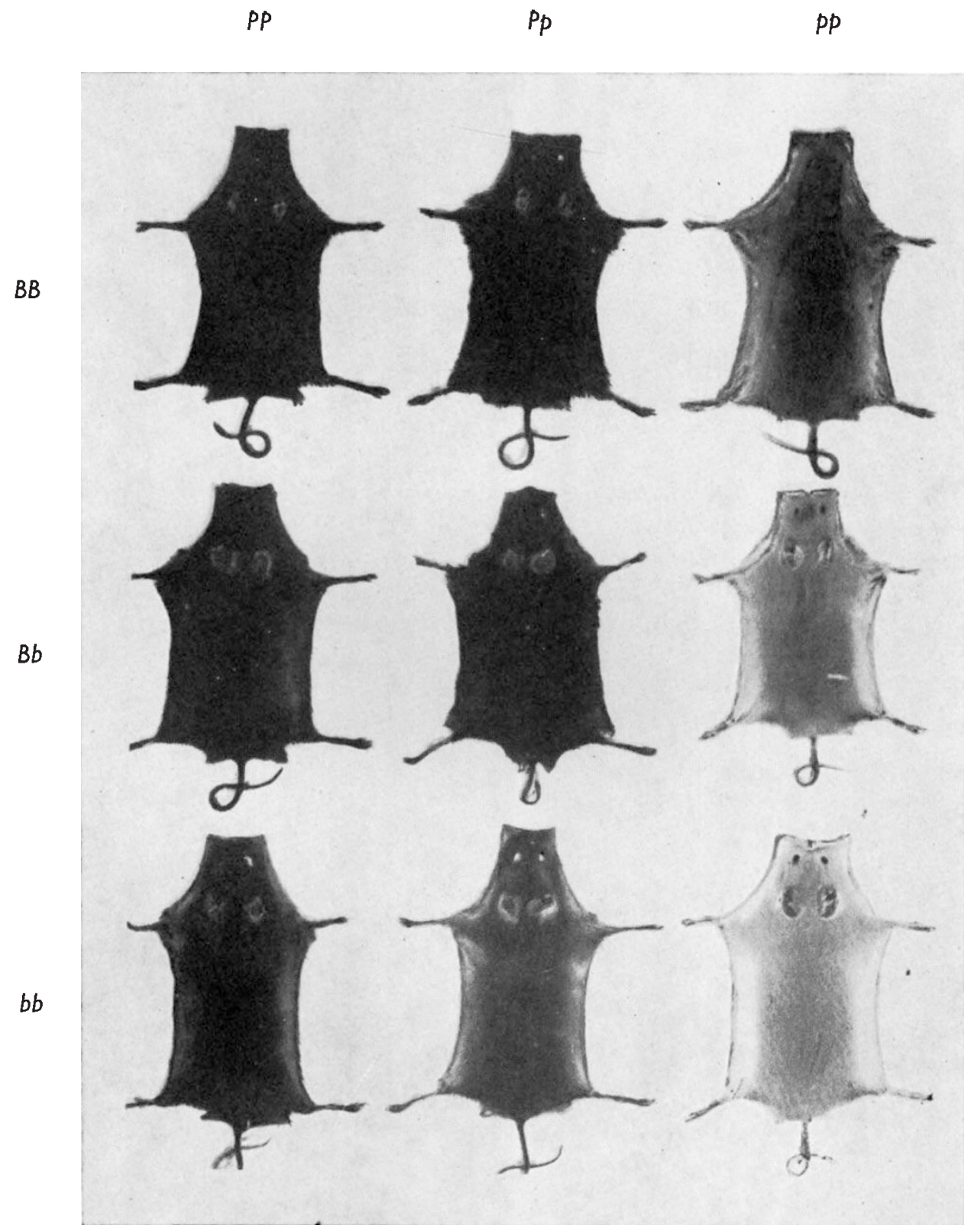

Plate I.-Mouse skins displayed to show mutual interaction of the $p$ and $b$ loci. $\quad \times \frac{1}{3}$. 